POSSIBILITY of SLOW EXTRACTION From the AGS BOOSTER

BOOSTER TECHNICAL NOTE

NO. 184

Y. Y. LEE

NOVEMBER 13, 1990

ALTERNATING GRADIENT SYNCHROTRON DEPARTMENT BROOKHAVEN NATIONAL LABORATORY

UPTON, NEW YORK 11973 


\title{
Possibility of Slow Extraction From the AGS Booster
}

\author{
Y. Y. Lee
}

\begin{abstract}
The possibility of slow extraction from the AGS Booster has been studied. It is possible to slow extract from the D-6 straight section with a thin wire septum located in the B-6 straight section which is presently not assigned. The extraction efficiency is estimated to be about $99 \%$.
\end{abstract}


When the AGS Booster was originally designed ${ }^{[1]}$, the possibility of slow extraction was not a consideration since the sole function of the machine was to boost the injection energy of the AGS both for protons and heavy ions. The Booster is, therefore, not an ideal synchrotron for slow extraction. Recently, however, a request has been made for possible use of slowly extracted beam from the Booster for nuclear physics as well as radio biology and radiation effects studies ${ }^{[2]}$.

Figure 1 shows the plan view of the Booster with the location of equipment to be installed ${ }^{[3]}$. The Booster has twelve long straight sections which are listed with present assignments in Table $I$.

Table I

\begin{tabular}{|c|l|l||}
\hline SUPERPERIOD & \multicolumn{1}{|c|}{ LOCATION 3 } & \multicolumn{1}{|c|}{ LOCATION 6 } \\
\hline A & RF (HI) & RF (P) \\
\hline B & RF (HI) & AVAILABLE \\
\hline C & INJECTION (HI) & INJECTION (P) \\
\hline D & DUMP KICKER ETC. & BEAM DUMP \\
\hline E & DAMPER KICKER ETC. & RF (P) \\
\hline F & EXTRACTION KICKER & EXTRACTION SEPTUM \\
\hline
\end{tabular}

As can be seen in the table, there is only one straight section available for whatever additional function the Booster may perform. Because of the very stringent high vacuum requirement, it is very difficult to place a water cooled current septum or its magnetic core lamination inside the vacuum chamber. Therefore, the extraction magnetic septum has to be isolated from the ring vacuum, and consequently the septum is rather quick-about 1 $\mathrm{cm}$. Therefore, we have to find an additional location for a thin electrostatic septum, which can be placed inside the vacuum chamber in order to have reasonably high extraction efficiency.

In this note we look into the possibility that the straight section D-6, where the beam dump is located, may be used for one of the extraction septa. Since the beam dump only occupies the inside aperture of the ring, the outside aperture can be used for the extraction septum.

We plan to use third integer resonance extraction, which is used at the AGS and proven to be very efficient. For the Booster we will extract at a horizontal tune of 4 and $2 / 3$ where the resonance is driven by $14^{\text {th }}$ harmonic of sextupoles. Since the Booster ring is rather congested with equipment, it is very hard to find a location for extra drive sextupoles. Therefore, we should utilize the existing lattice sextupoles in the ring. This 
means that the strength of the drive sextupoles is limited to the maximum field strength of the lattice sextupoles.

At the horizontal third integer resonance, the horizontal phase space grows in three different directions (or legs), which have a $120^{\circ}$ phase with respect to each other. The particle jumps from one leg to the next every turn, and comes back to original leg on the third turn where it is further away from the center than on the first pass. The increase in distance along the leg every third turn is called the spiral pitch.

In general, one wants the phase between the thin septum and the thick septum to be $90^{\circ}$ in order to have maximum spacial displacement for a given angular kick from the thin septum. In the case of third integer resonance, however, the ideal phase advance between septa is $60^{\circ}$ because of the other leg of growth. The phase between B-6 and D-6 is $560^{\circ}$ $(360+200)$ on the first encounter $(1 / 3$ turn), $2240(6 \times 360+80)$ degrees one turn later, and $3820(10 \times 360+320)$ degrees after another turn. The particles have to be ejected by this turn, otherwise the leg comes back to thin septum again. The phase advance at one and a third turn is $80^{\circ}$ which is acceptable though not ideal. We will use a one and a third turn extraction for the Booster.

Figure 2 shows the pattern of phase space growth at the thin electrostatic septum with drive sextupoles at A-2 and D-2 which produce all even harmonics. A slow orbit bump will bring the center of the closed orbit one inch away from the septum where particles inside the septum will be kicked 1.5 milliradian by the electrostatic force. On the next turn the kicked beam appears on the other leg toward inside of the vacuum chamber. The particles are extracted before the following turn so we do not see them on the third leg. Figure 3 shows the same phase space at the thick extractor septum. On the first encounter the kicked beam appears inside, but one turn later the beam appears at the septum about $1 \mathrm{~cm}$ away from closest part of the circulating beam. At this point the ejector magnet would extract the kicked beam.

An extraction efficiency of about $99 \%$ is estimated from the ratio of the electrostatic septum wire thickness $(1$ mil.) to the size of ejected beam. The spill stability or smoothness of the slow extracted beam is manly influenced by main magnet power supply and estimated to be about $4 \%$ modulation for every $10^{-4}$ variation of the main magnet supply. A spill servo system using the slow orbit bump may alleviate the problem since the spill modulation would be about $3 \%$ for every percent change in the slow orbit bump.

\section{References:}

[1] Accumulator/Booster Proposal for the AGS, BNL \# 32949-R, 1984.

[2] The Booster Applications Task Force Report, to be published, 1990.

[3] The AGS Booster Design Manual, 1988. 


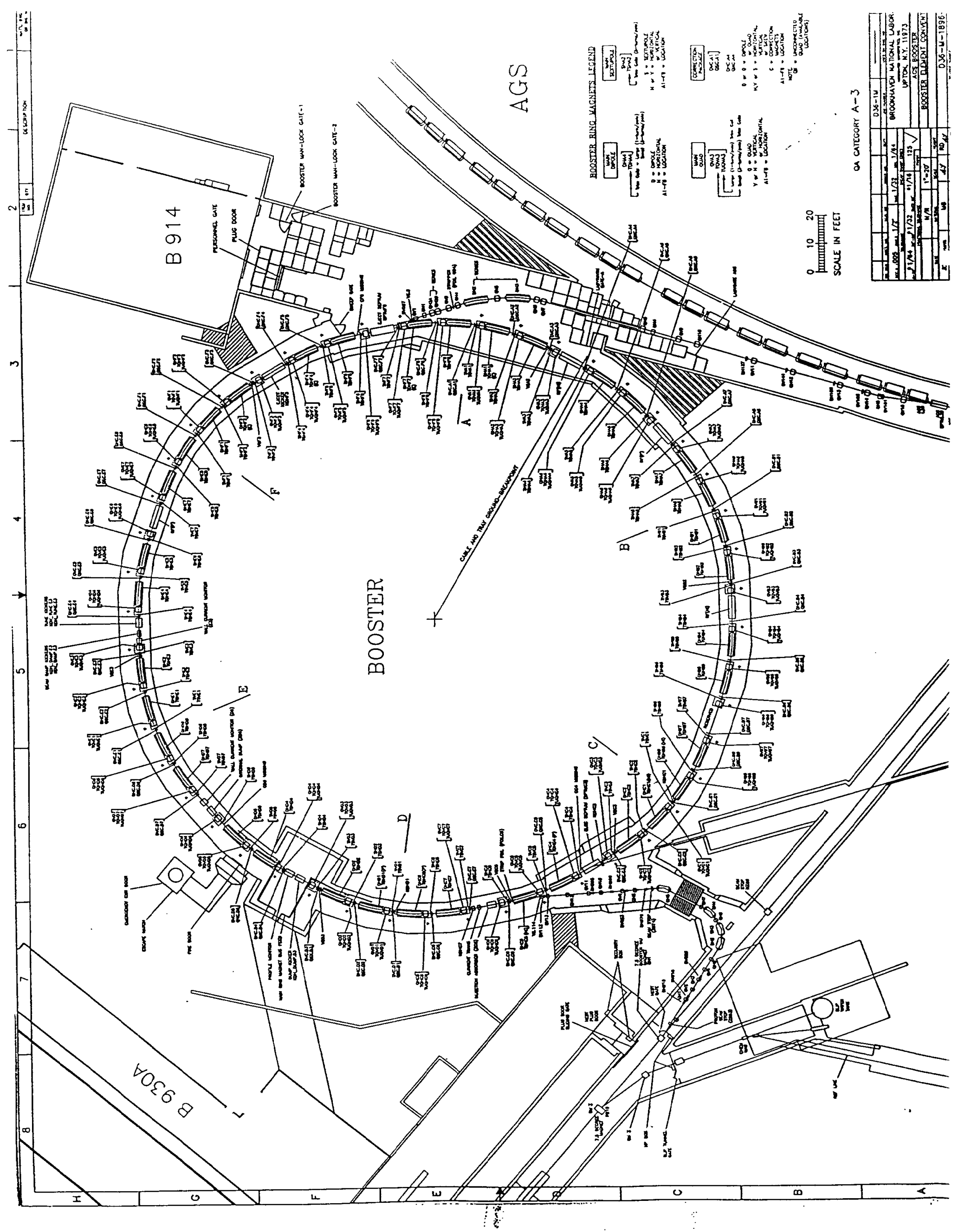




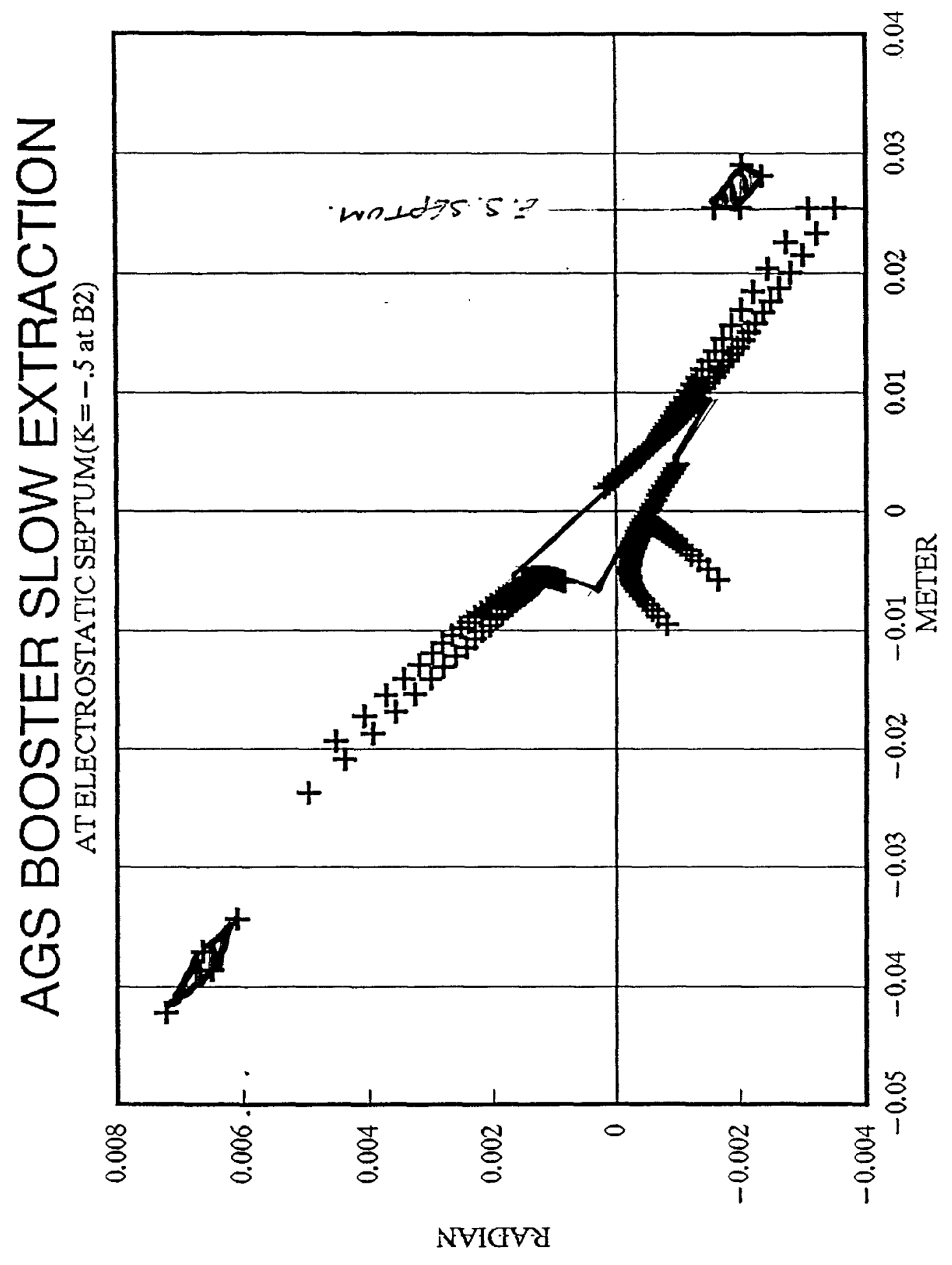




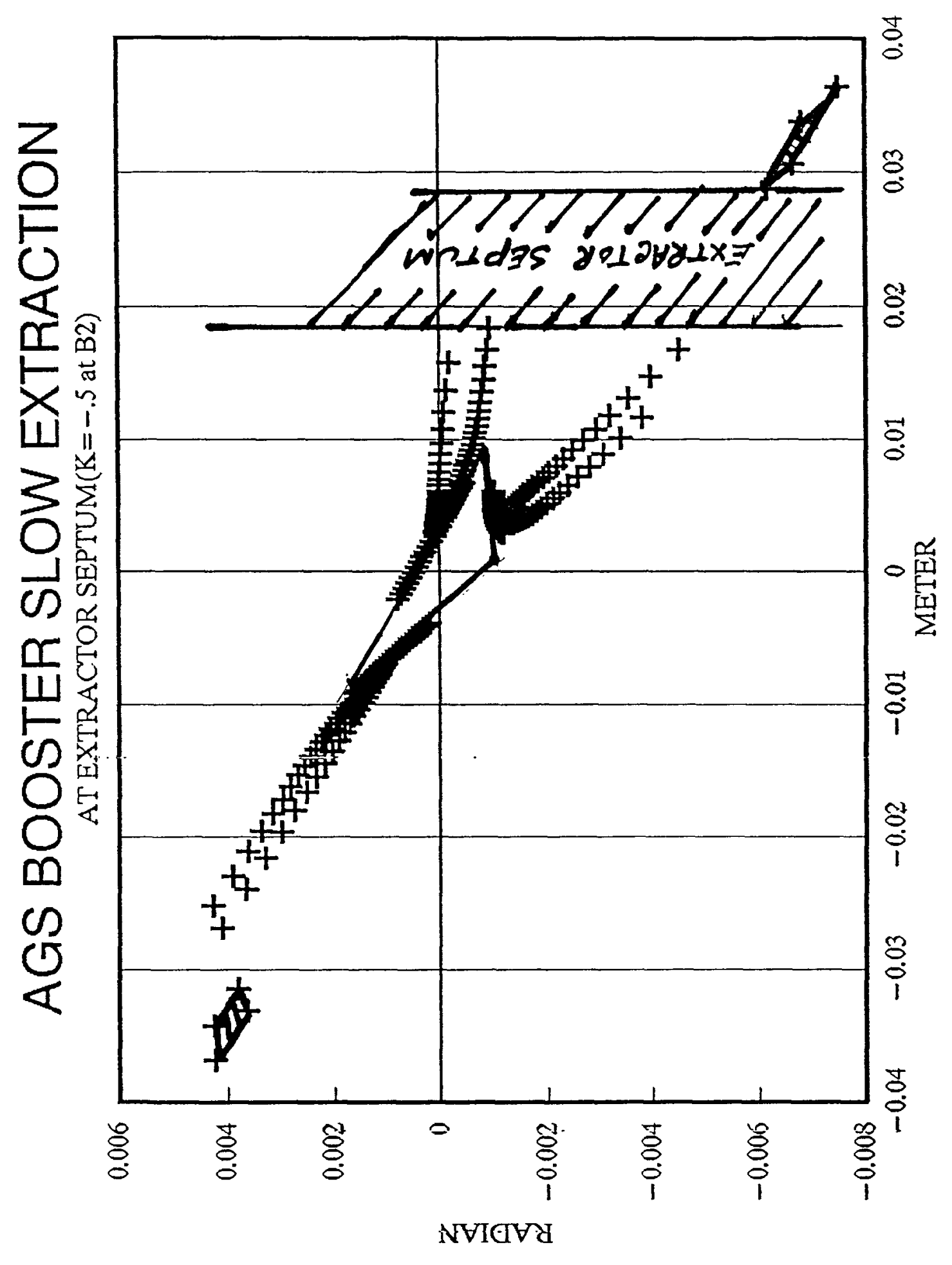

